\title{
APLICAÇÃO DA LÓGICA FUZZY E DO PROCESSO DE ANÁLISE HIERÁRQUICA NO DESENVOLVIMENTO DE UMA ARMADILHA ELÉTRICA SUSTENTÁVEL
}

\author{
Marcos dos Santos (marcosdossantos_doutorado_uff@yahoo.com.br) - Centro de \\ Análises de Sistemas Navais - Universidade Federal Fluminense \\ Viviane Viana Sofiste de Abreu (vsofiste@gmail.com) - Faculdade SENAI CETIQT \\ Carlos Francisco Simões Gomes (cfsg1@bol.com.br) - Universidade Federal Fluminense \\ Rubens Aguiar Walker (rubens.walker@gmail.com) - Universidade Federal Fluminense \\ Marcone Freitas dos Reis (marconefreis11@gmail.com) - Universidade Federal \\ Fluminense \\ Fabrício Baroni de Carvalho (baroni.fabricio@yahoo.com.br) - Centro Tecnológico do \\ Corpo de Fuzileiros Navais
}

\section{RESUMO}

A importância da tomada de decisão na organização é bastante clara e pode ser percebida empiricamente em qualquer análise organizacional. Porém, existe uma tendência de muitos gestores em confiar cegamente na sua capacidade de decidir com base na experiência ou na intuição, sem levar em conta informações e metodologias que lhes permitam ter muito mais clareza e eficácia naquilo sobre o que decidem. Diante deste cenário, esta pesquisa tem como objetivo aplicar a metodologia Analytic Hierarchy Process (AHP) combinada com a lógica Fuzzy para otimizar a tomada de decisão na seleção de um painel fotovoltaico na fase de concepção de projeto de uma armadilha elétrica. Para isto, a pesquisa se apoiou em metodologias de análise multicritério, mais especificamente no AHP e na lógica Fuzzy que é uma técnica que incorpora a forma humana de pensar. Ambas as metodologias são braços da pesquisa operacional. No intuito de demonstrar a aplicação desses dois métodos em um caso prático, foi elaborado um projeto conceitual de uma armadilha elétrica. Este produto utiliza para seu funcionamento energia fotovoltaica, que é a energia solar convertida através de painéis fotovoltaicos. Com intuito de otimizar a definição do painel fotovoltaico em função dos parâmetros de custo, vida útil, eficiência energética e classificação energética, foi aplicado um modelo baseado na metodologia AHP. Porém, como o parâmetro eficiência energética não possuía parâmetros numéricos evidentes, o modelo foi combinado com a metodologia Fuzzy afim de transformar o critério impreciso, nebuloso, em um critério mensurável, para aplicação do Método AHP. Como resultado, obtive-se a definição do painel mais adequado dentro dos parâmetros e requisitos levantados.

\section{Palavras chave: Lógica Fuzzy; AHP; Pesquisa Operacional; Teoria da Decisão; Engenharia do Produto}




\section{INTRODUÇÃO}

Os avanços tecnológicos, o processo de globalização, a preocupação com o meio ambiente e as mudanças governamentais na sociedade são fatores que estão influenciando cada vez mais os líderes e suas formas de decidir no ambiente das organizações. De acordo com Moritz e Pereira (2006), na atual sociedade do conhecimento, as empresas e seus respectivos líderes, que são capazes de se renovar continuamente através da inovação em estratégia, produtos, processos, relacionamento humano e conexão com a sociedade, definitivamente obterão amplas vantagens competitivas.

Neto (2007) destaca que existe uma tendência de muitos tomadores de decisão em confiar cegamente na sua capacidade de decidir com base na experiência ou na intuição, sem levar em conta informações e metodologias que lhes permitam ter muito mais clareza e eficácia naquilo sobre o que decidem. É evidente que, em muitos desses casos, o resultado pode ser adverso.

Souza (2010) e Portogente (2016) afirmam que, a Pesquisa Operacional é uma ciência aplicada voltada para a resolução de problemas reais. Tem como foco a aplicação de métodos científicos, matemáticos e estatísticos para resolução de problemas reais.

Conforme Souza et al (2010), a análise multicritério vem sendo utilizada como importante ferramenta quando se dispõe de vários critérios de análise, bem como quando se incorporam variáveis de difícil mensuração.

Segundo Souza (2004) apud Caneppele \& Seraphim (2010), "modelos matemáticos implantados em programas computacionais têm apresentado significativos resultados em sistemas não lineares e complexos nas mais diversas áreas [...]. Esses programas são capazes de resolver questões que os modelos clássicos, via de regra, não são capazes de fazê-lo. São os chamados de sistemas inteligentes, dentre os quais se destacam as Redes Neurais e a Lógica Fuzzy".

Considerando o contexto apresentado, esta pesquisa procura verificar se a aplicação de um modelo matemático que combina as metodologias AHP e a lógica Fuzzy é eficaz no processo de tomada de decisão para definição de um painel fotovoltáico para compor uma armadilha elétrica. Para isto, será apresentado um estudo de caso de um projeto conceitual de armadilha, com a aplicação das metodologias Fuzzy e AHP no intuito de definir o tipo de painel solar fotovoltaico que apresente a melhor relação custo x eficiência para o projeto proposto.

\section{FUNDAMENTAÇÃO TEÓRICA}

\subsection{O Método AHP Clássico (Analytic Hierarchy Process)}

As metodologias de análise multicritério permitem aos decisores compararem diversas alternativas analisando conjuntamente seus aspectos tangíveis, intangíveis, subjetivos e objetivos.

Existem diversas metodologias multicritério, porém, o AHP é uma das mais difundidas mundialmente. O método AHP foi criado nos Estados Unidos pelo professor Thomas L. Saaty (1980) na Wharton School of Business.

Segundo Saaty (1991) apud Neto (2007), esta teoria reflete o que parece ser um método natural de funcionamento da mente humana. [...]. Este modelo tenta simular a função cerebral humana, que agrupa elementos segundo suas propriedades comuns, criando um novo elemento em um nível superior de agregação. 
Para o autor, este processo de hierarquização auxilia o decisor tanto no entendimento de suas preferências e como estas o levaram a uma determinada escolha e consequentemente na documentação e justificação desse processo.

Segundo Gomes (2011), o Professor Saaty, observou que a percepção de estímulos por um indivíduo obedece a uma escala linear. Porém, além disto, existe também o chamado limite psicológico, que estabelece que o ser humano pode, no máximo, julgar corretamente $7 \pm 2$ pontos para distinguir essas diferenças. Assim, foi definida a Escala Fundamental que tem como objetivo traduzir em números as preferências do decisor, conforme a Tabela 1.

Tabela 1: Escala Fundamental de Saaty

\begin{tabular}{ccc}
\hline 1 & Igual Importância & $\begin{array}{c}\text { As duas atividades contribuem igualmente } \\
\text { para o objetivo }\end{array}$ \\
\hline 3 & $\begin{array}{c}\text { Importância pequena de } \\
\text { uma sobre a outra }\end{array}$ & $\begin{array}{c}\text { A experiência e o juízo favorecem uma } \\
\text { atividade em relação à outra }\end{array}$ \\
\hline 5 & $\begin{array}{c}\text { Importância grande ou } \\
\text { essencial }\end{array}$ & $\begin{array}{c}\text { A experiência e o juízo favorecem fortemente } \\
\text { uma atividade em relação à outra }\end{array}$ \\
\hline 7 & $\begin{array}{c}\text { Importância muito grande } \\
\text { ou demonstrada }\end{array}$ & $\begin{array}{c}\text { Uma atividade é muito fortemente favorecida } \\
\text { em relação à outra. Pode ser demonstrada na } \\
\text { prática. }\end{array}$ \\
\hline 9 & Importância absoluta & $\begin{array}{c}\text { relação à outra, com o mais alto grau de } \\
\text { segurança. }\end{array}$ \\
\hline $2,4,6,8$ & Valores Intermediários & $\begin{array}{c}\text { Quando se procura uma condição de } \\
\text { compromisso entre duas definições. }\end{array}$ \\
\hline
\end{tabular}

Fonte: Adaptado de (GOMES; ARAYA; CARIGNANO, 2011)

Quando uma atividade i em relação a uma atividade j recebe um dos valores da Tabela 1, a atividade j em relação i receberá o valor reciproco. Cada comparação par a par representa uma estimativa do coeficiente das prioridades de cada elemento. Dessa forma a comparação par a par de cada alternativa dentro de cada critério do nível imediatamente superior é realizada. A partir daí, o juízo verbal é convertido em uma escala de valores numéricos.

\subsection{A Lógica Fuzzy}

De acordo com Cosenza, Villela e More (2006), em 1965, o Professor Lotfi Zadeh formalizou o que, anos depois vinha a ser uma das maiores revoluções no setor matemático: a Lógica Fuzzy ou Lógica Nebulosa ou Lógica difusa. Esta teoria trata dos conjuntos não totalmente verdadeiros nem tampouco dos totalmente falsos. De acordo com Posselt et al (2009), a lógica clássica não abre espaço para valores intermediários. Já na lógica Fuzzy é possível analisar os valores intercessores. Em outras palavras, a lógica fuzzy deve ser vista como uma teoria matemática formal para a representação de incertezas.

Na figura 1 abaixo observa-se a diferença entre a lógica clássica e a lógica Fuzzy.

Figura 1: Diferença entre lógica clássica e lógica fuzzy. Fonte: (POSSELT et al., 2009)

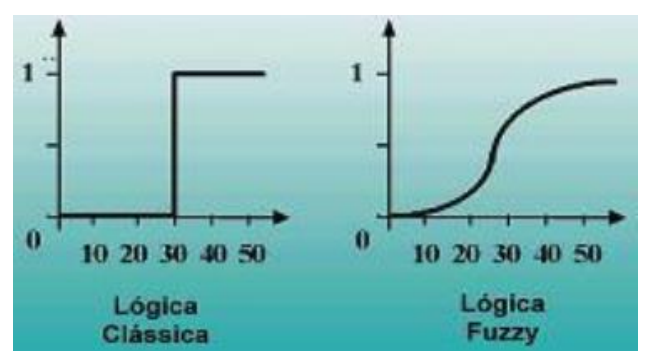


Segundo Posselt et al. (2009) a lógica clássica não abre espaço para valores intermediários. Já na lógica fuzzy é possível analisar os valores intercessores. Para JÚNIOR; NOVAKOWSKI (2005) APUD POSSELT et al. (2009), os conjuntos Fuzzy foram desenvolvidos para determinar o quanto um elemento pertence ou não a um determinado conjunto, com o uso de graus de pertinência $(\mu)$ que são valores no intervalo $[0 ; 1]$.

POSSELT et al.(2009) apresenta um exemplo de determinação de conjuntos Fuzzy. O item 1 da tabela 2 mostra a representação do universo. No item 2 são criados três conjuntos, sendo eles jovens, adultos e velhos. E, no item 3, são representados os graus de pertinência aos grupos em relação aos elementos do universo.

Tabela 2: Exemplo do uso de conjuntos Fuzzy.

1) $U=\{5,10,20,30,40,50,60,70\}$

2) Conjuntos difusos: Jovens, adultos, velhos

\begin{tabular}{cccl}
\hline Idades & Jovens & Adultos & Velhos $(\mu)$ \\
\hline 5 & 1,0 & 0,0 & 0,0 \\
10 & 1,0 & 0,0 & 0,0 \\
20 & 0,8 & 0,2 & 0,0 \\
30 & 0,5 & 0,4 & 0,1 \\
40 & 0,2 & 0,4 & 0,4 \\
50 & 0,1 & 0,3 & 0,6 \\
60 & 0,0 & 0,2 & 0,8 \\
70 & 0,0 & 0,0 & 1,0
\end{tabular}

Fonte: Adaptado de (POSSELT et al., 2009)

\section{ESTUDO DE CASO}

No intuito de demonstrar a aplicação da lógica Fuzzy combinada com o método AHP de tomada de decisão em um caso prático, foi elaborado um projeto conceitual de uma armadilha elétrica. Este produto utiliza para seu funcionamento energia fotovoltaica, que é a energia solar convertida através de painéis fotovoltaicos. Como no mercado existem muitas opções deste tipo de painel, foi aplicado um modelo que combina os dois métodos matemáticos citados anteriormente para realizar a seleção.

Considerando atributos como inovação, sustentabilidade, simplicidade e baixo custo, surgiu a ideia de um produto inovativo que utiliza energia solar das placas fotovoltaicas.

\subsection{O Produto}

A Erro! Fonte de referência não encontrada. 2 apresenta um esboço do produto. 
Figura 2. Esboço do dispositivo proposto movido à energia solar

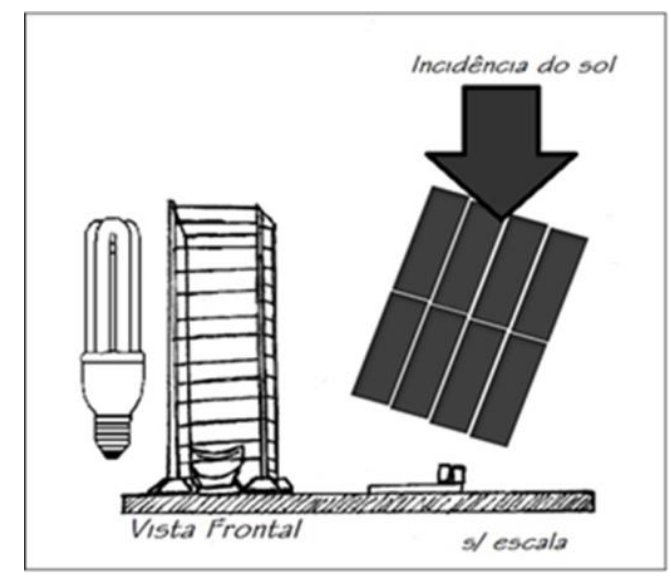

Fonte: Autores (2017)

Com o intuito de entender melhor a estrutura do produto proposto, a figura 3 apresenta a árvore do produto onde são detalhados todos os componentes da armadilha elétrica.

Figura 3. Árvores do Produto

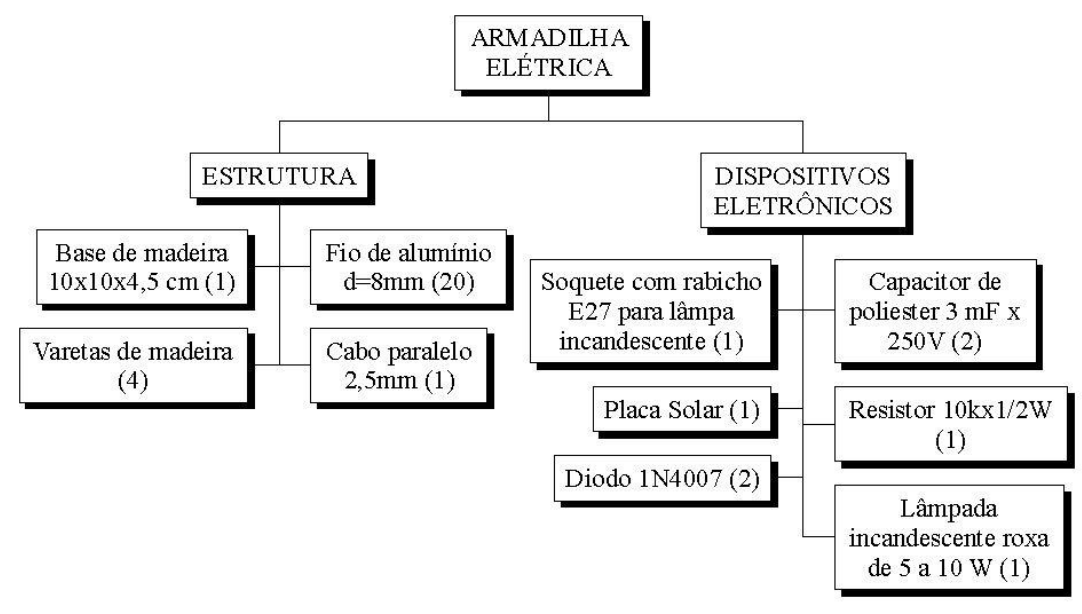

Fonte: Autores (2017)

O intuito é possibilitar à população a construção de um produto eficiente e de grande utilidade mesmo com pouco conhecimento técnico, e ao mesmo tempo incentivando a utilização de energia limpa e renovável.

\subsection{Seleção do Painel}

Através da tabela de consumo/eficiência energética para painéis fotovoltaicos homologados pelo Inmetro e publicadas em seu sítio (http://www.inmetro.gov.br/consumidor/pbe/tabela_fotovoltaico_modulo.pdf), foi possível verificar todos os módulos de painéis fotovoltaicos registrados e autorizados para fabricação, importação e comercialização no País.

Inicialmente, a tabela possuía 804 tipos de painéis, que, após a aplicação de alguns filtros, foram suprimidos todos os painéis cujas dimensões eram maiores que 60 centímetros; cujo peso era maior que 4 quilos; e aqueles cujos fornecedores encontravam-se à distâncias em relação ao ponto de origem (que foi fixado na cidade do Rio de Janeiro) que se encontraram fora do $1^{\circ}$ quartil. Desta forma, restaram então para análise 16 modelos de painéis. 
A partir dos modelos restantes, partiu-se para a análise da escolha do painel que represente a melhor relação custo $\mathrm{x}$ eficiência energética $\mathrm{x}$ vida útil $\mathrm{x}$ eficiência econômica através da combinação de duas metodologias: 1) A lógica $F u z z y$ e 2) o processo de análise hierárquica (AHP), demonstrados no desenvolvimento deste trabalho.

\subsection{Aplicação da lógica Fuzzy}

Para Green et al (2000), as eficiências energéticas dos painéis fotovoltaicos são classificadas em: Eficiência teórica, eficiência comercial e eficiência de laboratório. Este estudo toma como base a variação percentual entre as eficiências, para projetar nos painéis homologados pelos Inmetro suas eficiências teóricas e de laboratório. Os valores das eficiências estimadas podem ser verificados na figura 4 abaixo.

Figura 4. Painéis fotovoltaicos e respectivas eficiências energéticas. Fonte: Autores (2017)

\begin{tabular}{|c|c|c|c|c|c|c|c|c|}
\hline EMPRESA & MARCA & $\begin{array}{l}\text { MODELO / } \\
\text { CÓDIGO }\end{array}$ & MATERIAL & $\begin{array}{l}\text { PRODUÇÃO } \\
\text { MÉDIA DE } \\
\text { ENERGIA } \\
\text { (kWh/mês) }\end{array}$ & $\begin{array}{c}\text { EF. } \\
\text { TEÓRICA } \\
(\%)\end{array}$ & $\begin{array}{c}\text { EF. DE } \\
\text { LABORAT } \\
\text { ÓRIO }(\%)\end{array}$ & $\begin{array}{c}\text { EF. } \\
\text { COMERCI } \\
\text { AL }(\%)\end{array}$ & $\begin{array}{l}\text { CLASSIF. } \\
\text { ENERGÉTI } \\
\text { CA }\end{array}$ \\
\hline $\begin{array}{c}\text { KYOCERA SOLAR DO BRASIL } \\
\text { LTDA }\end{array}$ & KYOCERA & KS10T & Si-Poly & 1,25 & 1,60 & 6,11 & 9,4 & $E$ \\
\hline $\begin{array}{c}\text { KYOCERA SOLAR DO BRASIL } \\
\text { LTDA }\end{array}$ & KYOCERA & KS20T & Si-Poly & 2,50 & 1,88 & 7,15 & 11,0 & D \\
\hline $\begin{array}{c}\text { KYOCERA SOLAR DO BRASIL } \\
\text { LTDA }\end{array}$ & KYOCERA & KS5T & Si-Poly & 0,63 & 1,19 & 4,55 & 7,0 & $E$ \\
\hline $\begin{array}{c}\text { KYOCERA SOLAR DO BRASIL } \\
\text { LTDA }\end{array}$ & KYOCERA & SM-42KSM & Si-Poly & 5,25 & 2,10 & 8,00 & 12,3 & C \\
\hline $\begin{array}{c}\text { KYOCERA SOLAR DO BRASIL } \\
\text { LTDA }\end{array}$ & KYOCERA & SM-48KSM & Si-Poly & 6,00 & 2,13 & 8,13 & 12,5 & C \\
\hline $\begin{array}{l}\text { PRODUTOS ELETRONICOS } \\
\text { FRATA LTDA - EPP }\end{array}$ & $\begin{array}{l}\text { HETECH } \\
\text { ENERGY }\end{array}$ & $30 W-36 P$ & Si-Poly & 3,75 & 2,01 & 7,67 & 11,8 & D \\
\hline $\begin{array}{c}\text { SETIN \& STOYAN } \\
\text { EQUIPAMENTOS DE ENERGIA } \\
\text { SOLAR LTDA } \\
\end{array}$ & KOMAES & KM10 & Si-Poly & 1,25 & 1,57 & 5,98 & 9,2 & $E$ \\
\hline $\begin{array}{c}\text { SETIN \& STOYAN } \\
\text { EQUIPAMENTOS DE ENERGIA } \\
\text { SOLAR LTDA } \\
\end{array}$ & KOMAES & KM20 & Si-Poly & 2,50 & 1,83 & 6,96 & 10,7 & $E$ \\
\hline $\begin{array}{c}\text { SETIN \& STOYAN } \\
\text { EQUIPAMENTOS DE ENERGIA } \\
\text { SOLAR LTDA } \\
\end{array}$ & KOMAES & KM30 & Si-Poly & 3,75 & 1,93 & 7,35 & 11,3 & D \\
\hline $\begin{array}{c}\text { SETIN \& STOYAN } \\
\text { EQUIPAMENTOS DE ENERGIA } \\
\text { SOLAR LTDA } \\
\end{array}$ & KOMAES & KM5 & Si-Poly & 0,63 & 2,49 & 9,49 & 14,6 & A \\
\hline $\begin{array}{c}\text { SOLARTERRA IMPORTAÇÃO E E } \\
\text { COMÉRCIO DE EQUIPAMENTTOS } \\
\text { E SISTEMAS DE ENERGIA } \\
\text { ALTERNATIVA LTDA }\end{array}$ & $\begin{array}{c}\text { SOLARTERR } \\
\text { A }\end{array}$ & HG10 & Si-Mono & 1,38 & 1,83 & 8,55 & 9,5 & $E$ \\
\hline $\begin{array}{c}\text { SUN HOME ENERGIA SOLAR, } \\
\text { COMÉRCIO E IMPORTAÇÃO } \\
\text { LTDA }\end{array}$ & SUN HOME & ST-M10 & Si-Mono & 1,25 & 1,91 & 8,91 & 9,9 & $E$ \\
\hline $\begin{array}{c}\text { SUN HOME ENERGIA SOLAR, } \\
\text { COMÉRCIO E IMPORTAÇÃO } \\
\text { LTDA } \\
\end{array}$ & SUN HOME & ST-M30 & Si-Mono & 3,75 & 2,38 & 11,07 & 12,3 & C \\
\hline $\begin{array}{l}\text { YINGLI GREEN ENERGY DO } \\
\text { BRASIL S.A. }\end{array}$ & $\begin{array}{l}\text { YINGLI } \\
\text { SOLAR } \\
\end{array}$ & $\begin{array}{l}\text { YL010P- } \\
17 B 1 / 12 \\
\end{array}$ & Si-Poly & 1,25 & 1,72 & 6,57 & 10,1 & $E$ \\
\hline $\begin{array}{c}\text { YINGLI GREEN ENERGY DO } \\
\text { BRASIL S.A. }\end{array}$ & $\begin{array}{l}\text { YINGLI } \\
\text { SOLAR }\end{array}$ & $\begin{array}{l}\text { YL020P- } \\
17 \mathrm{~B} 1 / 6\end{array}$ & Si-Poly & 2,50 & 1,86 & 7,09 & 10,9 & $E$ \\
\hline $\begin{array}{l}\text { YINGLI GREEN ENERGY DO } \\
\text { BRASIL S.A. }\end{array}$ & $\begin{array}{l}\text { YINGLI } \\
\text { SOLAR }\end{array}$ & $\begin{array}{l}\text { YL030P- } \\
17 \mathrm{~B} 1 / 4\end{array}$ & Si-Poly & 3,75 & 1,88 & 7,15 & 11,0 & $E$ \\
\hline
\end{tabular}

De posse dos valores das eficiências teóricas, de laboratório e comercial para cada tipo de painel estudado, questionou-se qual seria a melhor eficiência para cada tipo de painel? O que pode ser considerado uma eficiência boa? Ou uma eficiência ruim? Ou uma eficiência moderada? Afim de encontrar respostas para estes questionamentos, buscou-se a aplicação da matemática nebulosa através de aplicação no software InFuzzy, cujo desenvolvimento metodológico será apresentado a seguir. 
Primeiramente foram definidas as variáveis de entrada e de saída, conforme figura 5.

Figura 5. Variáveis linguísticas de entrada e de saída. Fonte: Autores (2017)
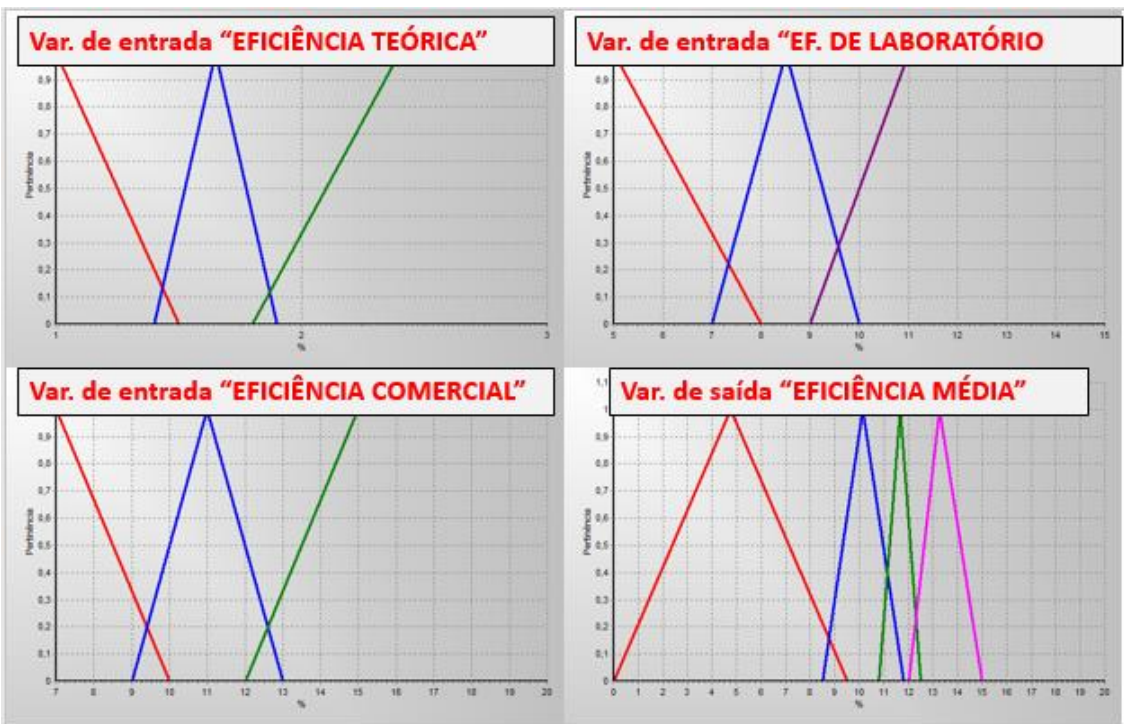

Para cada variável de entrada foram definidos três termos linguísticos: BAIXA, MODERADA E ALTA e para a variável de saída foram definidos quatro termos linguísticos: BAIXA, MODERADA, ALTA E MUITO ALTA. Os graus de pertinência para cada termo linguístico de cada uma das variáveis podem ser observados na figura 5. A próxima etapa foi a definição das regras de inferência, conforme figura 6 a seguir.

Figura 6. Bloco de regras do software InFuzzy Fonte: Autores (2017)

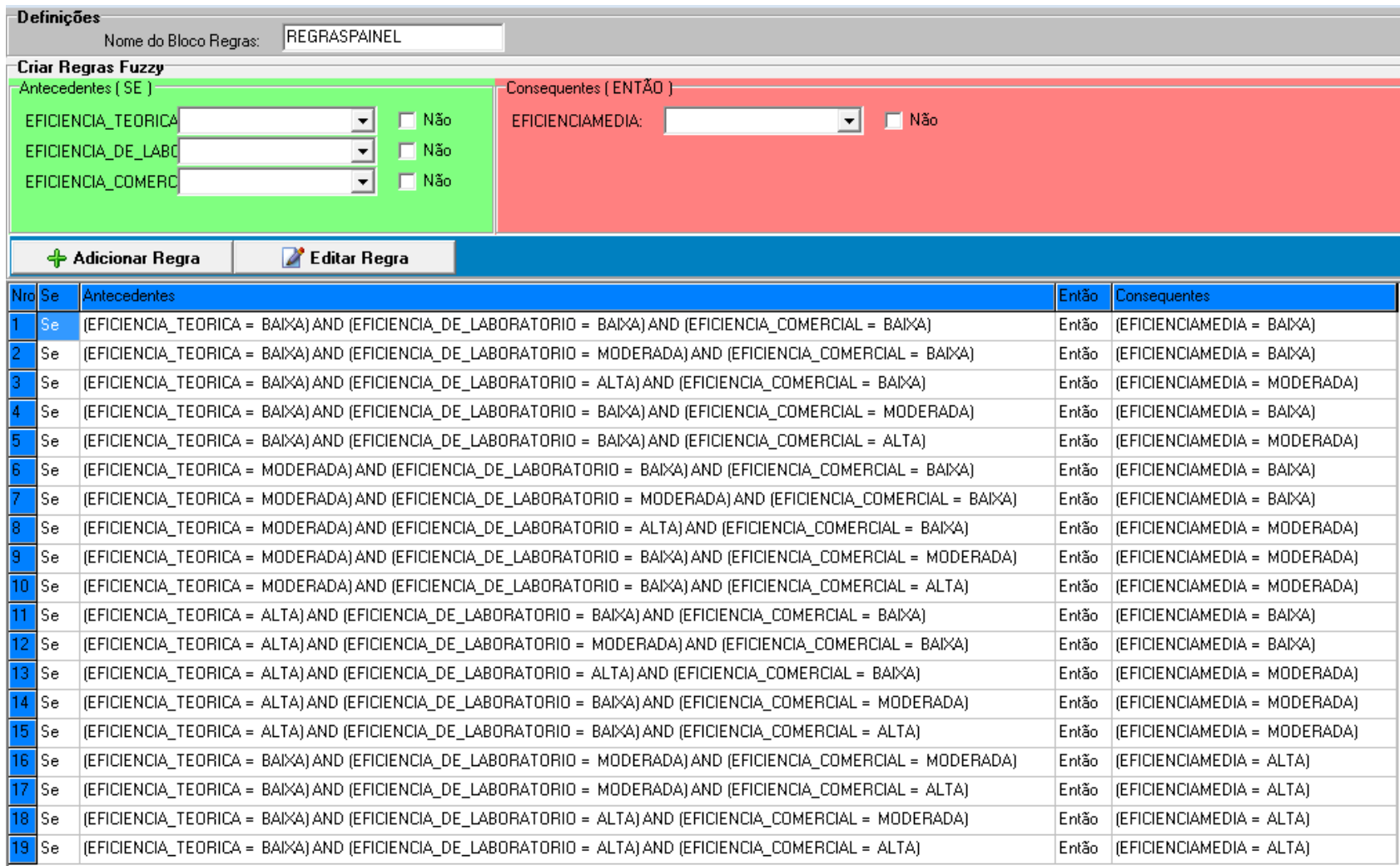

Com todas as variáveis e a regras de inferência definidas, partiu-se para a etapa de simulações. Criou-se 29 simulações com base nos dados disponíveis. Ao final da execução 
das simulações, o software gerou um valor de eficiência média e um gráfico de defuzzificação, com um valor de saída final para a variável "EFICIÊNCIAMEDIA". A defuzzificação pelo método do centro da gravidade, após a execução de todas as etapas, estabeleceu para a variável de saída, o valor de 10\%, conforme Erro! Fonte de referência não encontrada. 7 .

Figura 7. Bloco de regras do software InFuzzy Fonte: Autores (2017)

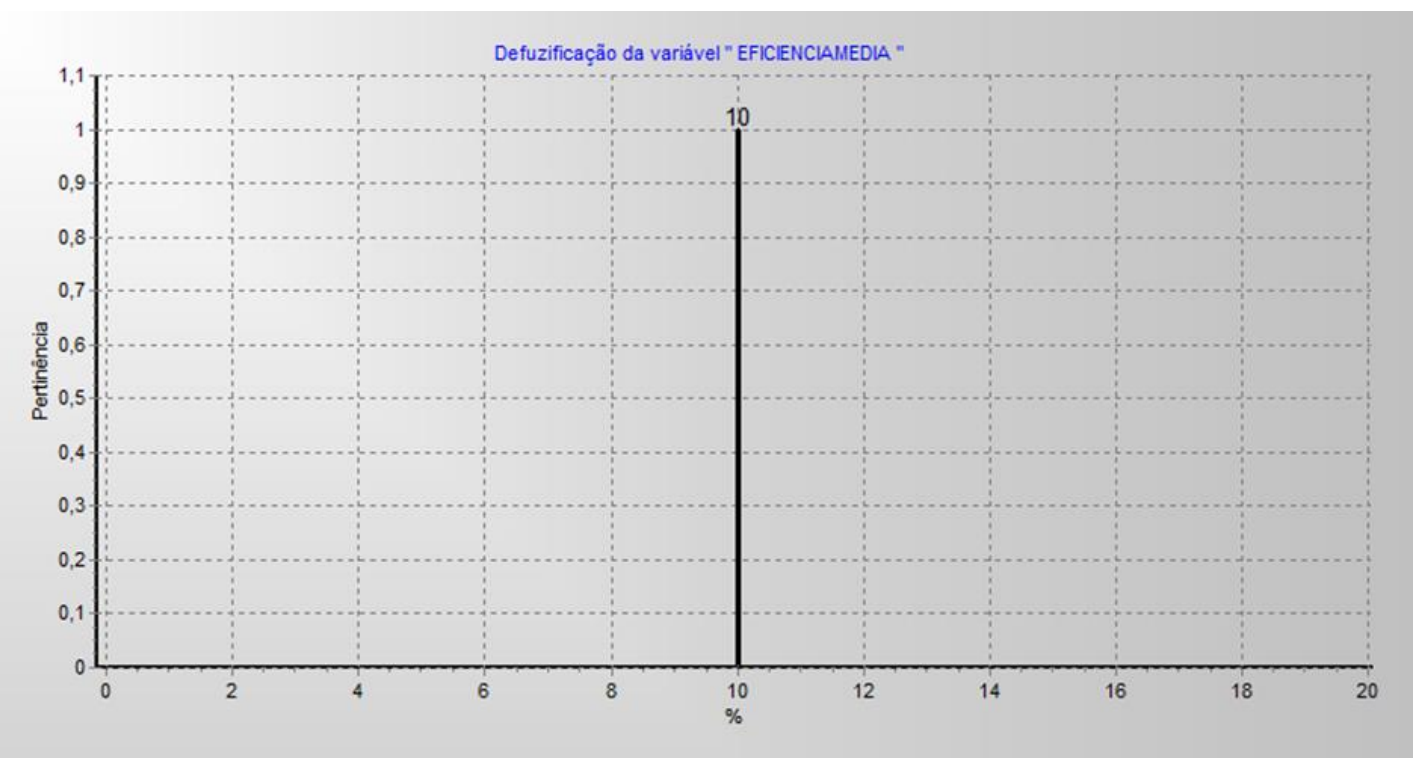

A eficiência média de $10 \%$ está inserida na característica de eficiência moderada, que representa valores de eficiência entre $8,5 \%$ e $12 \%$.

\subsection{A Aplicação do método AHP}

As premissas básicas adotadas para este projeto foram atributos como portabilidade, funcionamento, utilização de energia limpa, compromisso com o meio ambiente, facilidade de montagem e manuseio e custo acessível às populações menos favorecidas. Com base nelas, foram definidos os critérios para este estudo, que foram: (1) Eficiência Energética, (2) Classificação energética, (3) Vida Útil (Anos) e (4) Custo Total do painel.

A figura 8 a seguir mostra a matriz de decisão com os tipos de painéis a serem selecionados e os critérios de seleção adotados e também esta mesma matriz normalizada a fim de possibilitar a comparação inter-critérios.

Figura 8. Matrizes de decisão AHP. Fonte: Autores (2017) 


\begin{tabular}{|c|c|c|c|c|c|c|c|c|c|}
\hline \multicolumn{5}{|c|}{ MATRIZ DE DECISÃO } & \multicolumn{5}{|c|}{ MATRIZ DE DECISÃO NORMALIZADA } \\
\hline 20 & $\begin{array}{c}\text { Ef. } \\
\text { Energética } \\
(\%)\end{array}$ & \begin{tabular}{|c|} 
Class. \\
Energétic \\
$\mathrm{a}$
\end{tabular} & $\begin{array}{l}\text { Vida ùtil } \\
\text { (Anos) }\end{array}$ & $\begin{array}{l}\text { Custo } \\
\text { Total / } \\
\text { painél }\end{array}$ & & $\begin{array}{c}\text { Ef. } \\
\text { Energétic } \\
\text { a }(\%)\end{array}$ & $\begin{array}{c}\text { Class. } \\
\text { Energétic } \\
\text { a }\end{array}$ & $\begin{array}{l}\text { Vida ùtil } \\
\text { (Anos) }\end{array}$ & $\begin{array}{l}\text { Custo } \\
\text { Total/ } \\
\text { painél }\end{array}$ \\
\hline $\mathrm{KS} 20 \mathrm{~T}$ & 11,0 & 3,0 & 30,0 & $R \$ 346$ & $\mathrm{KS} 20 \mathrm{~T}$ & 0,09 & 0,08 & 0,09 & 0,08 \\
\hline SM-42KSM & 12,3 & 5,0 & 30,0 & $\mathrm{R} \$ 727$ & SM-42KSM & 0,10 & 0,13 & 0,09 & 0,04 \\
\hline SM-48KSM & 12,5 & 5,0 & 30,0 & $R \$ 831$ & SM-48KSM & 0,10 & 0,13 & 0,09 & 0,03 \\
\hline $30 \mathrm{~W}-36 \mathrm{P}$ & 11,8 & 3,0 & 30,0 & $\mathrm{R} \$ 520$ & $30 \mathrm{~W}-36 \mathrm{P}$ & 0,09 & 0,08 & 0,09 & 0,05 \\
\hline KM20 & 10,7 & 1,0 & 30,0 & R\$ 346 & KM20 & 0,08 & 0,03 & 0,09 & 0,08 \\
\hline KM30 & 11,3 & 3,0 & 30,0 & $R \$ 520$ & KM30 & 0,09 & 0,08 & 0,09 & 0,05 \\
\hline KM5 & 14,6 & 10,0 & 30,0 & $\mathrm{R} \$ 87$ & KM5 & 0,11 & 0,26 & 0,09 & 0,32 \\
\hline ST-M30 & 12,3 & 5,0 & 33,0 & $\mathrm{R} \$ 520$ & ST-M30 & 0,10 & 0,13 & 0,10 & 0,05 \\
\hline YL010P-17B 1/12 & 10,1 & 1,0 & 30,0 & $R \$ 173$ & YL010P-17B 1/12 & 0,08 & 0,03 & 0,09 & 0,16 \\
\hline YL020P-17B 1/6 & 10,9 & 1,0 & 30,0 & $R \$ 346$ & YL020P-17B 1/6 & 0,08 & 0,03 & 0,09 & 0,08 \\
\hline YL030P-17B 1/4 & 11,0 & 1,0 & 30,0 & $R \$ 520$ & YL030P-17B 1/4 & 0,09 & 0,03 & 0,09 & 0,05 \\
\hline$\sum_{\mathrm{si}=}$ & 128,50 & 38,00 & 333,00 & & $\sum_{\text {si }}=$ & 1,00 & 1,00 & 1,00 & 1,00 \\
\hline
\end{tabular}

Em seguida foi realizada a matriz de ponderações, através da classificação par a par entre os critérios estudados. Para classificação foi utilizada a escala fundamental de Saaty. A figura 9 a seguir mostra a matriz de ponderações, onde são comparados o grau de importância entre os critérios, e também esta mesma matriz normalizada a fim de possibilitar a comparação e obter com isto o vetor prioridade, que, ordenado do maior para o menor valor, representa o ranking dos critérios, que é o ranking de importância dos critérios elencados, conforme apresentado na figura 10 .

Figura 9. Matrizes de ponderações AHP. Fonte: Autores (2017)

\begin{tabular}{|c|c|c|c|c|c|c|c|c|c|c|}
\hline \multicolumn{5}{|c|}{ MATRIZ DE PONDERAÇÕES } & \multicolumn{6}{|c|}{ MATRIZ DE PONDERAÇÕES NORMALIZADA } \\
\hline & $\begin{array}{c}\text { EFICIÊNCIA } \\
\text { ENERGÉTICA }\end{array}$ & $\begin{array}{c}\text { CLASS. } \\
\text { ENERGÉTICA }\end{array}$ & $\begin{array}{l}\text { VIDA ÚTIL } \\
\text { (ANOS) }\end{array}$ & $\begin{array}{c}\text { Custo Total / } \\
\text { Painél }\end{array}$ & & $\begin{array}{c}\text { EFICIÊNCIA } \\
\text { ENERGÉTICA }\end{array}$ & $\begin{array}{c}\text { CLASS. } \\
\text { ENERGÉTICA }\end{array}$ & $\begin{array}{l}\text { VIDA ÚTIL } \\
\text { (ANOS) }\end{array}$ & $\begin{array}{c}\text { Custo Total / } \\
\text { Painél }\end{array}$ & $\begin{array}{c}\text { Vetor } \\
\text { prioridade }\end{array}$ \\
\hline $\begin{array}{c}\text { EFICIÊNCIA } \\
\text { ENERGÉTICA } \\
\text { VARIÁVEL FUZZY } \\
(\%)\end{array}$ & 1 & 3,00 & 5,00 & 2,00 & \begin{tabular}{|c|} 
EFICIÊNCIA \\
ENERGÉTICA \\
VARIÁVEL FUZZY \\
$(\%)$
\end{tabular} & 0,49 & 0,56 & 0,33 & 0,48 & 0,47 \\
\hline $\begin{array}{l}\text { CLASSIFICAÇÃO } \\
\text { ENERGÉTICA }\end{array}$ & 0,33 & 1 & 3,00 & 1,00 & $\begin{array}{c}\text { CLASSIFICAÇÃO } \\
\text { ENERGÉTICA }\end{array}$ & 0,16 & 0,19 & 0,20 & 0,24 & 0,20 \\
\hline $\begin{array}{c}\text { VIDA ÚTIL } \\
\text { (ANOS) }\end{array}$ & 0,20 & 0,33 & 1 & 0,17 & $\begin{array}{c}\text { VIDA ÚTIL } \\
\text { (ANOS) }\end{array}$ & 0,10 & 0,06 & 0,07 & 0,04 & 0,07 \\
\hline $\begin{array}{c}\begin{array}{c}\text { Custo Total / } \\
\text { Painél }\end{array} \\
\end{array}$ & 0,50 & 1,00 & 6,00 & 1 & $\begin{array}{c}\text { Custo Total / } \\
\text { painél }\end{array}$ & 0,25 & 0,19 & 0,40 & 0,24 & 0,27 \\
\hline$\sum_{a \mathrm{i}=}$ & 2,03 & 5,33 & 15,00 & 4,17 & $\sum_{\mathrm{ai}=}$ & 1 & 1 & 1 & 1 & 1 \\
\hline
\end{tabular}

Figura 10. Vetor prioridade dos critérios. Fonte: Autores (2017)

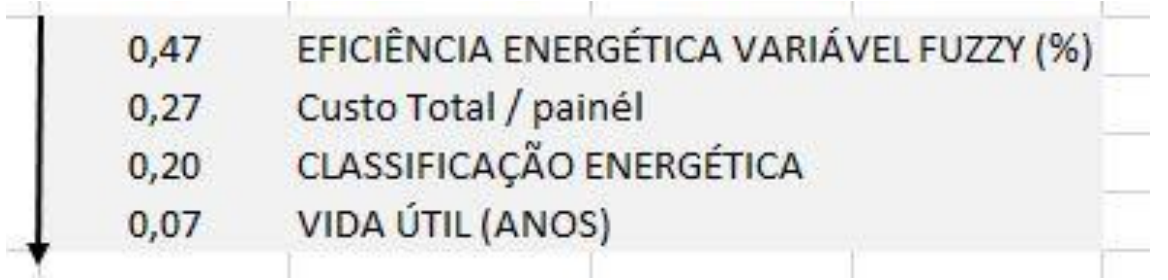

A próxima etapa foi a multiplicação entre a matriz de decisão normalizada e o vetor prioridade. Através do produto das matrizes, temos como resultado a matriz abaixo que representa o vetor prioridade das alternativas relacionado a cada painel que ele representa, conforme demonstrado na figura 11.

Figura 11. Vetor prioridade das alternativas. Fonte: Autores (2017) 


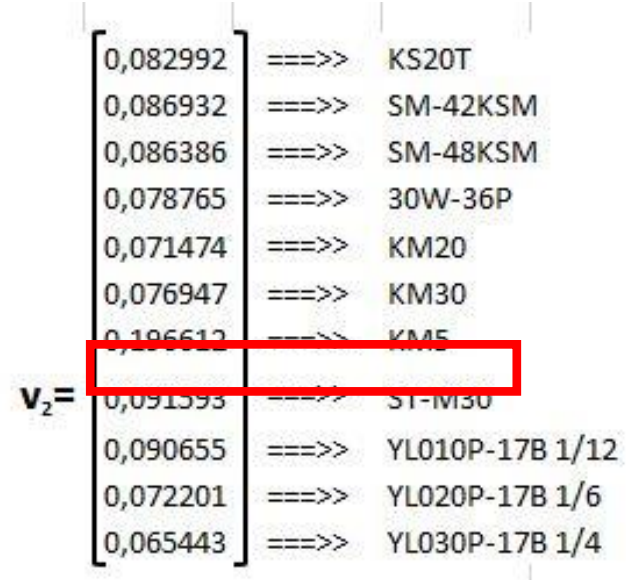

Conforme destacado na figura 11, o painel KM5 foi selecionado pois obteve o maior valor $(0,196612)$ entre os resultados do produto entre as matrizes.

\section{CONSIDERAÇÕES FINAIS}

O modelo matemático descrito no capítulo 3 se mostrou eficaz para alcançar o objetivo geral proposto pela pesquisa. A combinação das duas metodologias, a lógica Fuzzy e o AHP, possibilitou a conciliação dos critérios propostos e um refinamento dos resultados adquiridos.

Com o resultado obtido na lógica Fuzzy aplicado ao método AHP, foi possível a escolha do painel fotovoltaico modelo KM5 para a armadilha elétrica, dentre os 804 modelos homologados pelo Inmetro para comercialização no Brasil. Essa seleção não seria possível sem a combinação das duas metodologias.

Esta pesquisa comprovou que é possível trabalhar a visão sistêmica em detrimento à intuição, buscando minimizar a cegueira inatencional. A aplicação de modelos matemáticos possibilitou a escolha de um componente para compor um produto de forma sistêmica, não dependendo exclusivamente da intuição ou experiência para tomada de decisão.

\section{REFERÊNCIAS}

COSENZA, H.; VILLELA, L. E.; MORE, J. Aplicação de Um Modelo de Hierarquização Como Instrumento para Tomada de Decisão: Caso de uma Multinacional. XXVI Encontro Nacional de Engenharia de Produção - ENEGEP, p. 1-8, 2006.

COSTA NETO, Pedro Luiz de Oliveira. Qualidade e competência nas decisões, $1^{\circ}$ edição São Paulo: Blucher, 2007.

DE LIMA CANEPPELE, Fernando; SERAPHIM, Odivaldo José. APLICAÇÃO DA TEORIA FUZZY NO CONTROLE DE SISTEMAS DE GERAÇÃO DE ENER-GIAS ALTERNATIVAS. Energia na Agricultura, v. 25, n. 3, p. 24-41, 2010.

ENCONTRO REGIONAL DE ESTUDANTES DE MATEMÁTICA DO SUL, 16., 2010, Porto Alegre. APLICAÇÃO DO MÉTODO AHP-FUZZY. Porto Alegre - Rs: Editora Universitária da Pucrs, 2010. 12 p.

GOMES, Luiz Flavio A. M; ARAYA Marcela C. G., CARIGNAMO; Claudia. Tomada de decisão em cenários complexos: introdução aos métodos discretos do apoio multicritério à decisão, $1^{a}$ ed. São Paulo: Cengage Learning, 2011 
Moritz, Gilberto de Oliveira Processo decisório / Gilberto de Oliveira Moritz, Maurício Fernandes Pereira. - Florianópolis : SEAD/UFSC, 2006. 168p. "

PORTOGENTE. Disponível em:< https://portogente.com.br/portopedia/74372-pesquisaoperacional>. Acessado em 29/03/2017

POSSELT, E. L. et al. USO DE LÓGICA FUZZY PARA A OTIMIZAÇÃO DE SISTEMAS ENERGÉTICOS BASEADOS EM ENERGIA LIMPA. XXIX ENCONTRO NACIONAL DE ENGENHARIA DE PRODUÇÃO, p. 14, 2009. 